\title{
Measurement of Protein Turnover in Rat Liver
}

\author{
ANALYSIS OF THE COMPLEX CURVE FOR DECAY OF LABEL IN A \\ MIXTURE OF PROTEINS
}

\begin{abstract}
By PETER J. GARLICK, $\ddagger$ JOHN C. WATERLOW $\dagger$ and ROBERT W. SWICK*
* Department of Nutritional Sciences, University of Wisconsin, Madison, WI 53706, U.S.A., and $\dagger$ Department of Human Nutrition, London School of Hygiene and Tropical Medicine, Keppel Street, London WC1E 7HT, U.K.
\end{abstract}

(Received 20 January 1976)

\begin{abstract}
The curve for decay of ${ }^{14} \mathrm{C}$ in rat liver protein labelled by injection of $\mathrm{NaH}^{14} \mathrm{CO}_{3}$ was analysed to obtain the average turnover rate of mixed liver protein. Three different methods of analysis were used. (1) Unlike decay curves from homogeneous proteins, the curve did not fit a single exponential, but a good fit was obtained with three exponentials. By assuming that the mixture contained three major components with different turnover rates, the calculated value for the average turnover rate $(k)$ was close to $40 \%$ per day. (2) $k$ was also calculated from the area under the decay curve, a method which makes no assumptions about the number of proteins in the mixture. This method also gave a value close to $40 \%$ per day. (3) It was shown empirically, both by simulation of decay of label in model mixtures of protein and with the decay curve measured in vivo, that $k$ can be calculated from the time taken for the specific radioactivity to fall to $10 \%$ of its maximum value. This is an advantage, since the other two methods require the decay curve to be measured over a much longer period of time.
\end{abstract}

The rate of breakdown of a protein is usually measured by pulse labelling and observation of the subsequent rate of loss of label. With pure proteins the decay curve, plotted on semi-logarithmic axes, is generally close to a straight line whose gradient, in the absence of isotope recycling, is the fractional rate of degradation. If the protein is not homogeneous, however, the semi-log plot of radioactivity against time is not linear (e.g. Millward, 1970; Miehe, 1976). The gradient of the curve decreases with time, and it is not possible to obtain a unique value for the rate of breakdown of the mixture in the way that is used for pure proteins. Measurement of decay of label, which has been used to compare rates of turnover of cellular organelles (Arias et al., 1969) and changes in turnover brought about by dietary variation (e.g. Millward, 1970), therefore lacks precision, because the result is dependent on the duration of the experiment.

The decay curve of rat liver protein has been measured after labelling by injection of $\mathrm{NaH}^{14} \mathrm{CO}_{3}$, a precursor that has been shown to result in minimal re-utilization of isotope (Swick \& Ip, 1974). We have attempted to discover what useful information about the turnover of liver proteins can be derived from this

$\ddagger$ Present address: Clinical Nutrition and Metabolism Unit, Department of Human Nutrition, London School of Hygiene and Tropical Medicine, Keppel Street, London WC1E 7HT, U.K. curve. In particular we have proposed methods of estimating the mean rate of degradation in mixtures of protein. The results have already been reported in preliminary form (Garlick \& Millward, 1972; Garlick \& Swick, 1976).

\section{Methods}

We have analysed the decay curve obtained previously by Swick \& Ip (1974). Briefly, the experimental details are as follows. Female Holtzman rats weighing $230-250 \mathrm{~g}$ were housed individually in wire-bottomed cages and maintained at $23^{\circ} \mathrm{C}$ in a room with a $12 \mathrm{~h}$ light-dark cycle. Food $(12 \%$-casein diet; Chee \& Swick, 1976) and water were available ad libitum. The mean weight gain was less than $3 \%$ over the $6-$ week period. The rats were given $1 \mathrm{mCi}$ of $\mathrm{NaH}^{14} \mathrm{CO}_{3}$ $(50 \mathrm{Ci} / \mathrm{mol}$; Amersham $/$ Searle, Arlington Heights, IL, U.S.A.) by intraperitoneal injection, and killed in groups of four at intervals from $1 \mathrm{~h}$ to 41 days thereafter. Live proteins were prepared for radioactivity measurements as described by Swick \& Ip (1974). Radioactivity was measured by counting the samples in a Packard Tri-Carb liquid-scintillation counter with Aquasol (New England Nuclear Corp., Boston, MA, U.S.A.) as a scintillation mixture. Protein was determined (Lowry et al., 1951) by using bovine serum albumin as a standard. 


\section{Calculations}

\section{Turnover rates from curve-fitting data}

A standard multiple linear-regression program was kindly provided by Dr. Wayne Robarge (Institute of Environmental Studies, University of Wisconsin, Madison, WI, U.S.A.), who modified it to fit our data to two or more exponential components. Iteration was continued until the coefficients and exponents obtained would account for $100 \pm 5 \%$ of the total area under the curve and a reconstituted curve was within the experimental error of the data points.

The division of the decay curve into a number $(n)$ of exponentials implies that the mixture can be regarded as $n$ components with fractional rates of breakdown $\left(k_{i}, i=1, \ldots n\right)$ equal to the exponential rate constants $\left(\lambda_{i}\right)$. The values of the coefficients $\left(X_{i}\right)$ give the relative masses of the three components, but not directly. As Koch (1962) pointed out, the initial amount of label in a fraction will not be proportional only to its mass. If rates of synthesis and breakdown are equal, and if the label is given as a very short pulse, the initial labelling is also proportional to the turnover rate, i.e. $\mathrm{X}_{i}=\mathrm{C} m_{l} \lambda_{l}$, where $m_{i}$ is the mass of a protein and $C$ is a constant for all proteins labelled from the same pool of free amino acids. The relative mass of a fraction, as a proportion of the total mass, is then equal to:

$$
\left(\mathrm{X}_{i} / \lambda_{i}\right) / \sum_{i=1}^{n}\left(\mathrm{X}_{t} / \lambda_{i}\right)
$$

From the relative masses and the turnover rates of the components, the weighted mean fractional rate of breakdown $(k)$ can be calculated, i.e.:

$$
k=\sum_{i=1}^{n}\left(m_{i} k_{i}\right) / \sum_{i=1}^{n} m_{i}=\sum_{i=1}^{n} \mathrm{X}_{i} / \sum_{i=1}^{n}\left(\mathrm{X}_{i} / \lambda_{i}\right)
$$

\section{Mean turnover rate by stochastic analysis}

This method makes no assumption about the number of proteins in the mixture, but only that they are in the steady state and are labelled by a very short pulse which decays very rapidly after peak labelling of the protein.

If $B_{i}$ is the mass of a single protein broken down per unit time and $S_{t}$ is its specific radioactivity, the rate of loss of label from the protein is $\mathrm{B}_{t} \mathrm{~S}_{t}$. Therefore the total label lost from that protein between peak labelling (zero time) and infinite time equals $\int_{0}^{\infty} B_{i} S_{t} \mathrm{~d} t$, which is also equal to the initial amount of label in the protein. Hence, total label in protein mixture at zero time $\left(\mathbf{R}_{\mathbf{0}}\right)$ is given by

$$
\mathbf{R}_{0}=\int_{0}^{\infty}\left(\sum_{l=1}^{n} \mathbf{B}_{l} S_{l}\right) \mathrm{d} t
$$

In the steady state we assume that all $B_{\imath}$ values are constant with time and therefore:

$$
\mathbf{R}_{0}=\sum_{i=0}^{n} \mathbf{B}_{i} \mathbf{A}_{\boldsymbol{i}}
$$

where $A_{l}$ is the area under the decay curve for protein $i$ from zero time to infinity. However, it can be shown by integration of the precursor-product relationship of Zilversmit (1960) that if all proteins are labelled from the same precursor pool, all $A_{i}$ values are the same and equal to $A$, the area under the decay curve for the mixture. Hence, $R_{0}=A \cdot B$, where $B$ is the total mass of protein broken down in unit time. Dividing throughout by $M$, the total mass, and re-arranging to give $k(=\mathrm{B} / \mathrm{M})$ in terms of the initial (peak) specific radioactivity $\mathrm{S}_{0}, k=\mathrm{S}_{0} / A$.

\section{Evaluation of the area under the decay curve}

The simplest method is to plot the curve on linear graph paper, cut out the area and weigh it, but a mathematical approximation was preferred. It is assumed that the curve between two adjacent experimentally measured points (specific radioactivities $S_{1}$ and $S_{2}$ at times $t_{1}$ and $t_{2}$ ) can be approximated to a single exponential to calculate the area beneath it. It can readily be shown that the area beneath an exponential curve is equal to $\left(\mathrm{S}_{1}-\mathrm{S}_{2}\right)\left(t_{2}-t_{1}\right) / \ln \left(\mathrm{S}_{1} / \mathrm{S}_{2}\right)$. The total area under the curve is then calculated by summation of the areas under the separate sections.

For estimating the area under the tail of the curve, after the last measured point, it is assumed that the exponential appropriate to the last two points $\left(S_{n-1}, S_{n}\right)$ continues to infinite time. The area under the tail is therefore $S_{n}\left(t_{n}-t_{n-1}\right) / \ln \left(S_{n-1} / S_{n}\right)$.

\section{Simulation of decay of label in model protein mixtures}

The total label $\left(\mathbf{R}_{\boldsymbol{t}}\right)$ in a model mixture at different times after labelling by a pulse dose of tracer was calculated by assuming that initial labelling of each protein was proportional to its turnover rate, $k_{t}$ (Koch, 1962), that there was no re-utilization of label, and that decay of each protein was exponential. Hence:

$$
\mathrm{R}_{t}=\mathrm{C} \sum_{i=1}^{n}\left(m_{l} k_{i} \mathrm{e}^{-k_{i} t}\right)
$$

where $m_{t}$ is the mass of a single protein, and C is a constant. For a number of different time-points, the apparent turnover rate of the mixture $\left(k_{\mathrm{a}}\right)$ was calculated by assuming that the decay between zero time and that point was exponential, i.e. $\mathbf{R}_{t} / \mathbf{R}_{0}=\mathrm{e}^{-k_{a} t}$. A curve of $k_{\mathrm{a}}$ against the time-interval $(t)$ was plotted for each model mixture (Fig. 2). For the sake of comparison between curves, time was expressed in terms of the number of mean half-lives elapsed $\left(t / T_{t}\right)$ where $T_{\ddagger}=0.693 / k . k$ for each model can be calculated, 
since the masses and turnover rates of the components are known, i.e.:

$$
k=\sum_{i=1}^{n}\left(k_{i} m_{i}\right) / \sum_{i=1}^{n} m_{i}
$$

\section{Results}

\section{Decay of label in protein}

The complete decay curve of label in total liver protein over a 41-day period after injection of $\mathrm{NaH}^{14} \mathrm{CO}_{3}$ is shown in Fig. 1. Each point is the mean from four animals. Peak labelling occurred at approx. $1 \mathrm{~h}$, after which $60 \%$ of the label was lost in the first day. The subsequent decay was much slower, but by 41 days, less than $0.3 \%$ of the label remained. The change in gradient with time is illustrated if the apparent turnover rate is calculated on the assumption that decay between two time-points is exponential, the method that is used for purified proteins. The decay between peak labelling $(1 \mathrm{~h})$ and 1 day gives an apparent turnover rate of $77 \%$ /day, whereas that between peak labelling and 30 days gives $17 \%$ /day. This method is therefore useless for obtaining the mean turnover rate of total liver protein. However, it is logical to assume that the reason why the curve is not a single exponential is because of the heterogeneity of turnover rates of liver proteins, and that the curve might therefore be better described by a number of exponential terms. The following three analyses of the curve are based on this assumption.

\section{Three-exponential fit}

The experimental data were fitted to the following expression as described in the Calculations section:

$$
\text { Specific radioactivity }=\sum_{i=1}^{n}\left(\mathrm{X}_{t} \mathrm{e}^{-\lambda_{t^{t}}}\right)
$$

A three-exponential expression $(n=3)$ gave a somewhat better fit than a two-exponential $(n=2)$. Because, when $n=3$, the reconstituted curve fits the experimental data so well, it seemed unlikely that the addition of more components would give a better resolution of the curve (see Fig. 1). The values of $X_{t}$ and $\lambda_{t}$ for the three exponentials are shown in Table 1 .

The fact that the curve approximates to a threeexponential one suggests that the protein mixture can be regarded as three groups of proteins whose masses and average turnover rates can be derived from the values of $X_{t}$ and $\lambda_{t}$. This is clearly an arbitrary division of liver proteins, which exhibit a wide spectrum of turnover rates (Schimke, 1970). The choice of three exponentials rather than four or more is governed more by the degree of precision in the data than by any correspondence to physiological reality. As shown in the Calculations section, the three values of $\lambda_{t}$ are the fractional turnover rates of the three fractions and the relative masses are given by the three values of $X_{i} / \lambda_{l}$. These are also given in Table 1 .

Once the relative masses and turnover rates are known, the mean fractional rate of turnover $(k)$ can also be calculated (see the Calculations section). From the decay-curve data in Table $1, k$ for liver is $41.3 \% /$ day.

\section{Stochastic method}

We also tried another way of estimating $k$ from the decay curve, by a method which requires no assump-

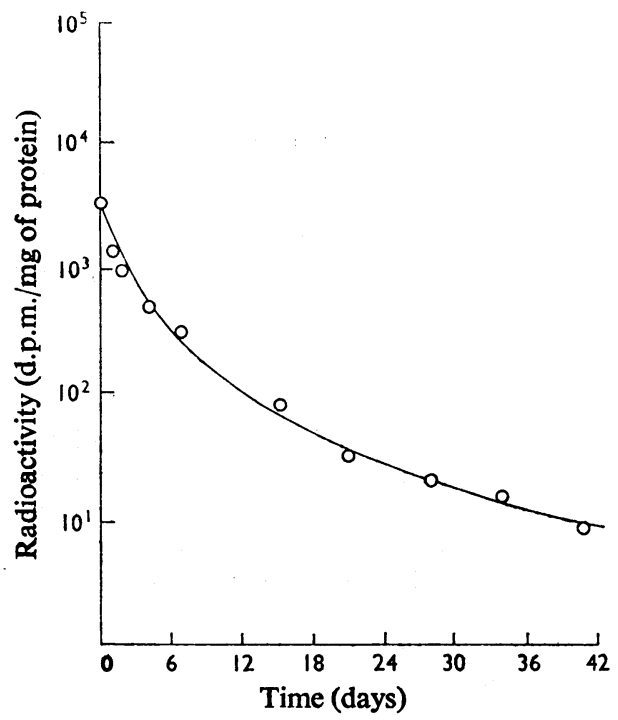

Fig. 1. Decay of specific radioactivity of liver proteins labelled by injection of $\mathrm{NaH}^{14} \mathrm{CO}_{3}$, taken from Swick \& Ip (1974)

Each point is the mean from four rats and the S.E. values averaged $5.8 \%$ of the mean. The line is drawn from the three-exponential expression, whose parameters are contained in Table 1.

Table 1. Parameters of the three-exponential curve derived by computer analysis of the decay of tracer in liver protein

The three exponentials were derived from the curve shown in Fig. 1 as described in the Calculations section. $X_{t}$ are the proportions (as \%) of the initial specific radioactivity apportioned to each component, $\lambda_{t}$ are the fractional rates of turnover of the components and $X_{t} / \lambda_{t}$ are their relative masses, expressed as a percentage of the total mass.

\begin{tabular}{crcr} 
Component & $\mathrm{X}_{t}$ & \multicolumn{1}{c}{$\lambda_{t}$} & $\mathrm{X}_{t} / \lambda_{t}$ \\
1 & 52.4 & 2.28 & 9.5 \\
2 & 44.0 & 0.274 & 66.4 \\
3 & 3.6 & 0.0619 & 24.1
\end{tabular}


Table 2. Relative masses and turnover rates $(k)$ of the components in the model protein mixtures used to construct the curves of Fig. 2

In model 1 the proteins are present in equal amounts, and turnover rates cover a fairly wide range. In model 2 proteins are in unequal amounts, with a similar range of turnover rates, and in model 3 the disproportions are exaggerated. Model 4 is a condensed form of the list of turnover rates of liver enzymes given by Schimke (1970). The liver was assumed to contain unit quantity of each enzyme in the list (e.g. there were four enzymes with turnover rates close to 1.09 days $^{-1}$ ). $k$, the mean turnover rate of the mixture, was calculated as described in the Calculations section.

\begin{tabular}{|c|c|c|c|c|c|}
\hline Model 1 & Relative mass & $k\left(\right.$ days $\left.^{-1}\right)$ & Model 4 & Relative mass & $k\left(\right.$ days $\left.^{-1}\right)$ \\
\hline & $\begin{array}{l}1 \\
1 \\
1 \\
1 \\
1 \\
1 \\
1 \\
1 \\
1\end{array}$ & $\begin{array}{r}0.693 \\
0.460 \\
0.350 \\
0.173 \\
0.116 \\
0.087 \\
0.069 \\
0.046 \\
0.035 \\
k=0.225\end{array}$ & & $\begin{array}{l}1 \\
1 \\
2 \\
4 \\
5 \\
6 \\
3 \\
5 \\
1\end{array}$ & $\begin{array}{r}16.632 \\
8.316 \\
5.544 \\
1.090 \\
0.749 \\
0.324 \\
0.205 \\
0.165 \\
0.048 \\
k=1.699\end{array}$ \\
\hline Model 2 & $\begin{array}{c}\text { Relative mass } \\
1 \\
5 \\
10\end{array}$ & $\begin{array}{r}\left.k \text { (days }^{-1}\right) \\
1.00 \\
0.20 \\
0.04 \\
k=0.15\end{array}$ & Model 3 & $\begin{array}{c}\text { Relative mass } \\
1 \\
10\end{array}$ & $\begin{array}{r}\left.k \text { (days }^{-1}\right) \\
10 \\
0.1 \\
k=1.0\end{array}$ \\
\hline
\end{tabular}

tion about the number of proteins in the mixture and does not require the use of a computer. In the steady state, $k$ is given by the maximum specific radioactivity divided by the area under the decay curve between zero and infinite time (see the Calculations section). The value of $k$ obtained by this method from the decay curve shown in Fig. 1 is $42.8 \%$ /day, which is in close agreement with the result obtained by analysis into exponentials.

The problem with this method is to allow for the part of the area after the last point at $\mathbf{4 1}$ days. Its magnitude was estimated by assuming that the decay between the last two measured points was continued to infinity as a single exponential. This showed that the area under the tail was only about $1.5 \%$ of the total area. Indeed, only $5 \%$ of the total area remained after 21 days had elapsed. The period of 21 days therefore represents the minimum interval over which measurements must be made to obtain a reasonably accurate estimate of $k$. It would probably be justifiable to neglect the area under the tail, because it is likely to represent mainly label that has been reutilized (Heath \& Barton, 1973). Although labelling the protein with $\left[{ }^{14} \mathrm{C}\right]$ carbonate results in minimal re-utilization, a small amount of recycling is unavoidable.

\section{$k$ from decay over short periods}

It was shown above that the apparent rate of turnover measured by assuming exponential decay between peak labelling and 1 day was $77 \%$ /day, greater than the value of $k$ obtained by analysis of the full curve. Similarly measurements made over a period of 30 days underestimate the value of $k$. Hence there must be a point on the decay curve at which the apparent rate equals $k$. We have investigated the possibility that this point can be predicted to the required degree of accuracy. For this we have simulated the decay of total label in a number of model mixtures of protein containing various proportions of proteins with different rates of turnover. The composition of these models and their calculated mean rates of turnover are shown in Table 2. For each model the apparent rate of turnover $\left(k_{\mathrm{a}}\right)$ was plotted against the timeinterval over which it was measured (Fig. 2). $k_{\mathrm{a}}$ was calculated by assuming that decay between zero time and a later point was exponential, equivalent to drawing a straight line on a decay curve (on semi-log axes) between the initial point and a later point and measuring its gradient. The notable feature of the curves shown in Fig. 2 is that in spite of the considerable variation in $k_{\mathrm{a}}$ with time, the point where $k_{\mathrm{a}}=k$, indicated on each curve by a triangle, is at an interval of three to four times the mean half-life of the mixture. We can find no theoretical reason why this should be so, but it has been true for all other models that we have tested, when these bear any similarity to the type of mixture that we expect in living tissues.

In practical terms, since we do not already know the mean half-life of the mixture, measurement to the point where the specific radioactivity has decayed to 


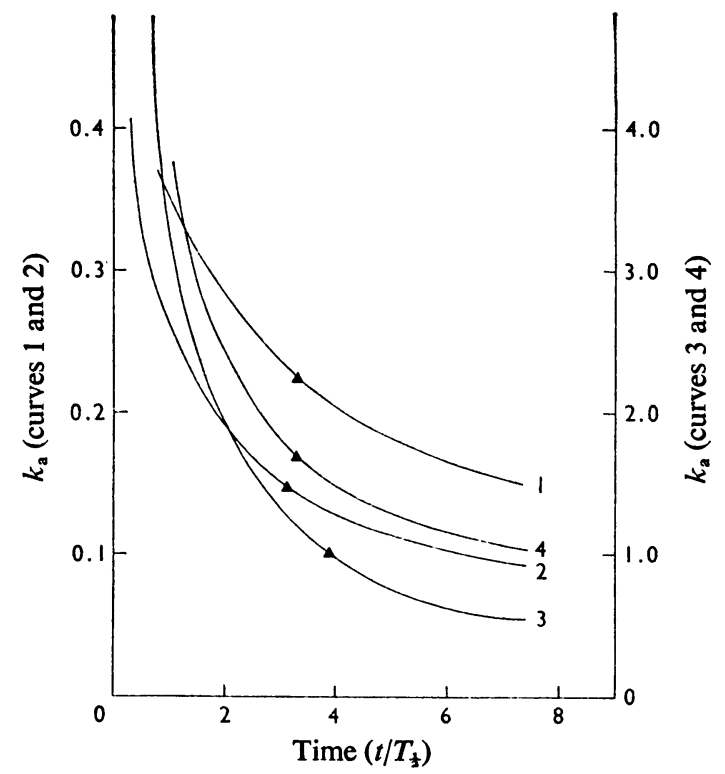

Fig. 2. Curves derived by simulation of the decay of label in the model protein mixtures given in Table 2

The method of constructing the curves is given in the Calculations section. It was assumed that the initial specific radioactivity of each protein was proportional to its turnover rate, and the points on the decay curve for the mixture were then calculated. The apparent turnover rate of the mixture $\left(k_{\mathrm{a}}\right)$ was determined from the gradient of the line drawn between the initial point and a later point $(t)$ on the curve, on the assumption that decay between these two points was exponential. The graph shows the values of $k_{\mathrm{a}}$ corresponding to different periods of measurement $(t)$ for each model. $t$ was expressed in terms of the number of mean half-lives elapsed $\left(t / T_{\xi}\right)$. The mean half-lives $\left(T_{t}\right)$ of the mixtures were calculated from the average protein turnover rates $(k)$ given in Table 2 . Separate curves are shown for models $1,2,3$ and 4 , and the triangles ( $\Delta$ ) show the position on each curve where $k_{\mathrm{a}}=k$.

$10 \%$ of its initial (peak) value should give a reasonable estimate of $k$. In Fig. 1, the decay curve for liver protein in vivo, $10 \%$ of peak labelling occurs after 6 days and gives a value for $k$ of $40 \% /$ day. This method therefore is able to give an estimate of the mean rate of turnover with both simulated decay of label in model mixtures of protein and decay of labelled liver protein in vivo. Further, the time-interval required for measurement of $k$ for liver protein is only 6 days, compared with a minimum of 21 days for the more complete analyses presented above.

\section{Discussion}

The three methods we have used to overcome the non-exponential loss of isotope from protein mix- tures caused by heterogeneity of turnover rates rely on the same set of assumptions, which were made clear in the Calculations section. These are that a steady state is maintained throughout the experiment, with the turnover rates of the proteins remaining constant, and that there is no recycling of isotope. Except when the protein mass is changing very rapidly, recycling of isotope is likely to be the most important. This has been minimized by the use of $\mathrm{NaH}^{14} \mathrm{CO}_{3}$ to label protein (Millward, 1970; Swick \& Ip, 1974; Ip et al., 1974; Ip \& Harper, 1975). Although the three methods all give the same result, a better check on their credibility is to compare the results obtained here with those obtained by a different method, in this case by incorporation of labelled amino acids (Table 3 ). In the steady state, the rate of synthesis equals the rate of breakdown; incorporation and decay measurements should then give the same result. The values in Table 3 show that the results obtained from the decay curve are in the middle of the range of results from incorporation experiments. Reasons for discrepancies include: (i) any recycling of label in the decay experiment would result in an underestimate of $k$; (ii) an error in the incorporation experiments caused by heterogeneity of protein turnover rates; this error is likely to be small if the experiment is short in duration (Koch, 1962; Garlick \& Millward, 1972); (iii) uncertainty about the site and specific radioactivity of the free amino acid during incorporation. In experiments in which we believe this problem is not serious (e.g. Swick, 1958; Fern \& Garlick, 1974), there is fairly good agreement with the results from decay.

In addition, the results from all methods will be influenced to some extent by the synthesis of plasma proteins in the liver. With the decay curve their influence will be very small, since they leave the liver shortly after synthesis $(16 \mathrm{~min}$ for albumin; Peters \& Peters, 1972) and the measurements of decay only begin after $1 \mathrm{~h}$. The measurements of incorporation used for comparison have been obtained from experiments lasting a few hours or more, and the contribution of plasma proteins to the estimates of liver protein synthesis will also be small.

Curves for incorporation of radioisotope into liver proteins have also been analysed to give fractions with different turnover rates (Buchanan, 1961; Richmond et al., 1963). Koch (1962) re-processed the data of Swick (1958) for incorporation of $\left[{ }^{14} \mathrm{C}\right]$ arginine into liver protein during continuous feeding of $\left[{ }^{14} \mathrm{C}\right]$ carbonate. He concluded that a number of interpretations were possible to explain the observed change in apparent turnover rate from $29.3 \%$ /day to $18.6 \% /$ day between 1 day and 8 days of exposure to the radioisotope; (i) that $83 \%$ of liver proteins turn over at a rate of $38.6 \% /$ day and that the remainder are stable; or (ii) that there is an almost continuous spectrum of rates of turnover which are better expressed by a distribution function. The three fractions that we 
Table 3. Comparison of the estimates of the average rate of turnover of rat liver protein obtained by analysis of the decay curve with those from measurement of incorporation of labelled amino acids into protein

\begin{tabular}{|c|c|c|}
\hline Method & Reference & $\begin{array}{c}\text { Turnover rate } \\
\text { (\%/day) }\end{array}$ \\
\hline Decay curve after ${ }^{14} \mathrm{CO}_{3}{ }^{2-}$ & & \\
\hline $\begin{array}{l}\text { Three exponentials } \\
\text { Stochastic } \\
10 \% \text { peak labelling }\end{array}$ & - & $\begin{array}{l}41.3 \\
42.8 \\
40.0\end{array}$ \\
\hline $\begin{array}{l}\text { Incorporation of labelled } \\
{\left[{ }^{14} \mathrm{C}\right] \mathrm{Tyrosine} \text { infusion }} \\
{ }^{14} \mathrm{CO}{ }^{2-} \text { feeding } \\
{\left[{ }^{14} \mathrm{C}\right] \text { Lysine injection }} \\
{\left[{ }^{14} \mathrm{C}\right] \text { Lysine infusion }} \\
{\left[{ }^{14} \mathrm{C}\right] \text { Glycine infusion }}\end{array}$ & $\begin{array}{l}\text { Garlick et al. }(1973,1975) \\
\text { Swick (1958) } \\
\text { Haider \& Tarver (1969) } \\
\text { Waterlow \& Stephen (1968) } \\
\text { Fern \& Garlick (1974) }\end{array}$ & $\begin{array}{c}42-67 \\
30 \\
41 \\
77 \\
36\end{array}$ \\
\hline
\end{tabular}

have obtained from the decay curve represent yet another way of describing the turnover of liver proteins, which also fits the data used by Koch (1962). A simulation (similar to the one used to construct the curves of Fig. 2) of the labelling of the three protein fractions given in Table 1 during exposure to a precursor of constant specific radioactivity predicts an apparent turnover rate at 1 day of $30.0 \%$ /day and at 8 days of $18.9 \%$ /day. These values are almost identical with the data used by Koch (1962) in the analysis described above.

It is noteworthy that the estimate of the turnover rate of the largest fraction gives a half-life of 2.5 days for this component (Table 1). This value is not unlike that obtained in most experiments using $\mathrm{NaH}^{14} \mathrm{CO}_{3}$ when the disappearance of radioactivity is measured between day 1 and days 4-6, an interval frequently used in such studies. The component turning over fastest will have gone through three half-lives before the first measurement is made, and thus has been overlooked in most experiments. The size of the third component is surprising, but may be largely discounted because the greatest error is encountered in its estimation. To estimate this component more accurately, it would have been necessary to define the tail of the curve with more data points over a longer period of time.

Of the three methods that we have used, division into exponentials gives most information about the turnover of liver protein. Although the fractions derived are a somewhat arbitrary division of liver proteins according to their turnover rates, in cultured fibroblasts the breakdown process has been shown to differentiate between 'fast' and 'slow'-turning-over proteins (Poole, 1975). However, the method needs the use of a computer, and in situations where it is the mean rate of breakdown that is required the stochastic method will be found to be more convenient.

The method of measuring the decay to $10 \%$ of peak labelling was devised because the full analysis by either of the other methods needs an accurate decay curve to be measured over an inconveniently long time-period, 21 days at least. All the methods assume that a steady state is maintained, and in many cases this would not be possible for such long time-periods. Only a few days are required for decay to $10 \%$ of peak radioactivity to occur. Although this method was derived empirically, it has provided an estimate of the mean turnover rate of a protein mixture which agrees with that obtained by other methods. For comparisons of mean turnover rates, for example in different cellular organelles or in tissues of animals given diets of different composition, measurement to $10 \%$ of peak radioactivity is more suitable than conventional methods in which decay is measured over an arbitrary time-interval.

This research was supported jointly by the United States Public Health Service (grant no. AM 14704), the University of Wisconsin Graduate School and the Medical Research Council, U.K.

\section{References}

Arias, I. M., Doyle, D. \& Schimke, R. T. (1969) J. Biol. Chem. 244, 3303-3315

Buchanan, D. L. (1961) Arch. Biochem. Biophys. 94, 500-511

Chee, P. Y. \& Swick, R. W. (1976) J. Biol. Chem. 251, 1029-1034

Fern, E. B. \& Garlick, P. J. (1974) Biochem. J. 142, 413419

Garlick, P. J. \& Millward, D. J. (1972) Proc. Nutr. Soc. 31, 249-255

Garlick, P. J. \& Swick, R. W. (1976) Proc. Int. Symp. Intracellular Protein Catabolism 2 nd in the press

Garlick, P. J., Millward, D. J. \& James, W. P. T. (1973) Biochem. J. 136, 935-946

Garlick, P. J., Millward, D. J., James, W. P. T. \& Waterlow, J. C. (1975) Biochim. Biophys. Acta 414, 71-84 
Haider, M. \& Tarver, H. (1969) J. Nutr. 99, 433-445

Heath, D. F. \& Barton, R. N. (1973) Biochem. J. 136, 503-518

Ip, C. \& Harper, A. E. (1975) J. Nutr. 105, 885-893

Ip, M. M., Chee, P. Y. \& Swick, R. W. (1974) Biochim. Biophys. Acta 354, 29-38

Koch, A. L. (1962) J. Theor. Biol. 3, 283-303

Lowry, O. H., Rosebrough, N. J., Farr, A. L. \& Randall, R. J. (1951) J. Biol. Chem. 193, 265-275

Miehe, M. (1976) Proc. Int. Symp. Intracellular Protein Catabolism 1st in the press

Millward, D. J. (1970) Clin. Sci. 39, 591-603

Peters, T., Jr. \& Peters, J. C. (1972) J. Biol. Chem. 247, 3858-3863
Poole, B. (1975) in Intracellular Protein Turnover (Schimke, R. T. \& Katunuma, N., eds.), pp. 249-264, Academic Press, New York

Richmond, J. E., Shoemaker, W. C. \& Elwyn, D. H. (1963) Am. J. Physiol. 205, 848-856

Schimke, R. T. (1970) in Mammalian Protein Metabolism (Munro, H. N., ed.), vol. 4, pp. 178-228, Academic Press, New York and London

Swick, R. W. (1958) J. Biol. Chem. 231, 751-764

Swick, R. W. \& Ip, M. M. (1974) J. Biol. Chem. 249, 6836-6841

Waterlow, J. C. \& Stephen, J. M. L. (1968) Clin. Sci. 35, 287-305

Zilversmit, D. B. (1960) Am. J. Med. 29, 832-848 